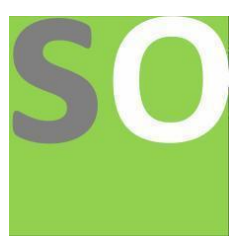

Article title: The distribution of primes in a short interval

Authors: Jan Feliksiak[1]

Affiliations: N.A.[1]

Orcid ids: $0000-0002-9388-1470[1]$

Contact e-mail: jan.feliksiak1@yahoo.com

License information: This work has been published open access under Creative Commons Attribution License $\mathrm{http}: / / c r e a t i v e c o m m o n s . o r g / l i c e n s e s / b y / 4.0 /$, which permits unrestricted use, distribution, and reproduction in any medium, provided the original work is properly cited. Conditions, terms of use and publishing policy can be found at https://www.scienceopen.com/.

Preprint statement: This article is a preprint and has not been peer-reviewed, under consideration and submitted to ScienceOpen Preprints for open peer review.

DOI: 10.14293/S2199-1006.1.SOR-.PP3QFL7.v1

Preprint first posted online: 12 July 2021

Keywords: Density of primes in a short interval, Distribution of primes, P. Erdos conjecture for primes in short intervals, Logarithmic integral, Maximal prime gaps bounds, Maximal prime gaps Supremum, Prime Gap Merit, Prime Number Theorem, Second Cramer's conjecture, Second Hardy-Littlewood conjecture 


\title{
THE DISTRIBUTION OF PRIMES IN A SHORT INTERVAL
}

\author{
JAN FELIKSIAK
}

\begin{abstract}
.
This research paper begins the presentation, with the topic of the distribution of primes in a short interval. The lower and upper limits for the number of primes within the interval are defined unambiguously. This provides us with a solid foundation, to resolve conclusively the Second Hardy - Littlewood $\pi_{k} \geq \pi_{(n+k)}-\pi_{n}$ conjecture. The paper concludes with the Merit of a Prime Gap and the Second Harald Cramér's Conjecture.
\end{abstract}

2010 Mathematics Subject Classification. 0102,1102, 1103, 11A41, 11L20, 11N05, 11N37.

Key words and phrases. Density of primes in short interval, distribution of primes, Erdös P. conjecture for primes in short intervals, logarithmic integral, maximal prime gaps lower bound, maximal prime gaps Supremum, Prime Gap Merit, Prime Number Theorem, Second Cramér's conjecture, Second Hardy and Littlewood's conjecture, Supremum for primes in short intervals, Tailored logarithmic integral, the primorial. 


\section{Contents}

1. Preliminaries 2

2. The distribution of primes in short interval $\mathcal{G} s_{(n)} \quad 2$

2.1. Preliminaries 2

2.2. The Maximum bound for the number of primes in $\mathcal{G} s_{(n)} \quad 4$

2.3. Historical reflection. Density of primes in a short interval 7

2.4. The Second Hardy and Littlewood's conjecture 8

2.5. The Merit of a Prime Gap and Harald Cramér's Conjecture 11

References $\quad 13$

3. Appendix 16

\section{Preliminaries}

Within the scope of the paper, prime gap of the size $\mathfrak{g} \in \mathbb{N} \mid \mathfrak{g} \geq 2$ is defined as an interval between two primes $p_{n}, p_{(n+1)}$, containing $(\mathfrak{g}-1)$ composite integers. Maximal prime gap of the size $\mathfrak{g}$, is a gap strictly exceeding in size any preceding gap. All calculations and graphing were carried out with the aid of the Mathematica ${ }^{\circledR}$ software. For all $n \in \mathbb{N} \mid n \geq 8$, we make the following definitions:

Definition 1.1 (Maximal Prime Gap).

$\mathfrak{g}=p_{(n+1)}-p_{n}$

Some graphs in this paper implement a variant of logarithmic scaling of the horizontal axis given by:

Definition 1.2 (Scaling factor). $\xi=\frac{\log _{10}\left(\frac{n}{24}\right)}{\log _{10}(24)}$

The definitions 1.3 and 1.4 are stated in a relaxed form, whereby the Floor in $\mathcal{G} s_{(n)}$ had been dropped:

Definition 1.3 (Interval length). $c=\mathcal{G} s_{(n)}=5\left(\log _{10} n\right)^{2}-\frac{15}{8}\left(\log _{10} n\right)$

Definition 1.4 (Interval endpoint). $t=(n+c)$

2. The Distribution OF PRIMES IN ShORT INTERVAL $\mathcal{G} s_{(n)}$

\subsection{Preliminaries.}

The main result of this section is the Maximum bound for the number of primes within the interval $\mathcal{G}_{(n)}$. First we define several related concepts, in accordance with the definition 1.3 of $\mathcal{G} s_{(n)}$ and 1.4 of the interval end-point $t$. Now, by Theorem 3.1 we have that:

$$
\pi_{(t)}>\pi_{(n)} \quad \forall n \in \mathbb{N} \mid n \geq 11
$$

Theorem 3.18 states that, the estimation error of the tailored logarithmic integral $T L i_{(n)}$ at every step exceeds the value of the inverse of the pertinent prime number 

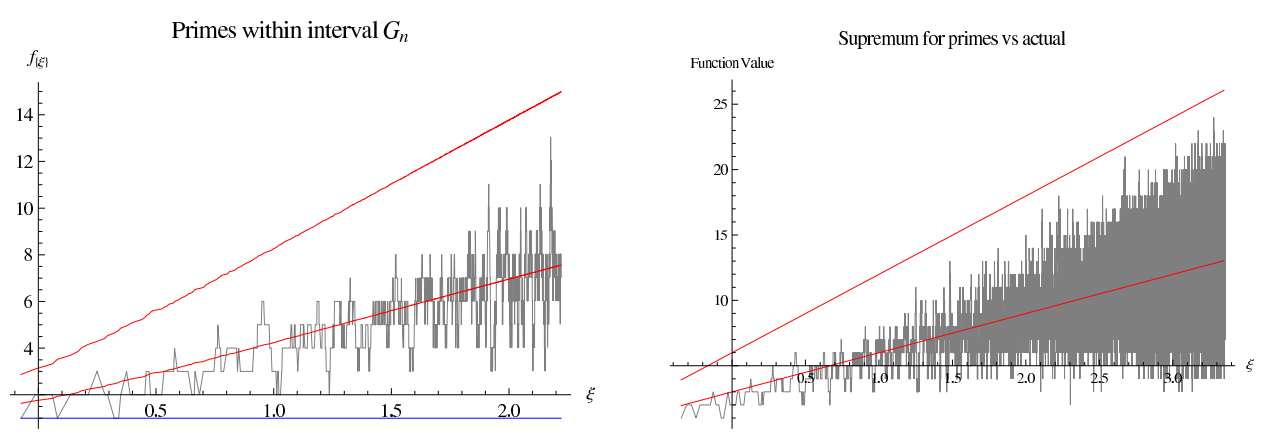

FIGURE 1. shows the graphs of the actual counts of the number of primes within the interval $\mathcal{G} s_{(n)}$ (Grey), the Maximum and the average bounds (red), as well as the Infimum bound (Blue). The graphs are drawn with respect to $\xi$ at every $n \in \mathbb{N}$ within the range, which corresponds to $8 \leq n \leq 3000$ in the left figure, and $8 \leq n \leq 10^{6}$ in the right figure.

for all $p_{i} \in \mathbb{N} \mid p_{i} \geq 13$ :

$$
\left(\int_{\theta_{1}}^{\theta_{2}} \frac{d t}{\log t}\right)-1>\frac{1}{p_{(i+1)}}
$$

where $p_{(i+1)}$ is associated with the upper limit of integration $\theta_{2}$. Consequently, over the length of the interval $\mathcal{G}_{s_{(n)}}$ we obtain:

$$
\sum_{n<p_{i+1} \leq t}\left[\left(\int_{\theta_{1}}^{\theta_{2}} \frac{d t}{\log t}\right)-1\right]>\sum_{n<p \leq t} \frac{1}{p} \quad \forall n \in \mathbb{N} \mid n \geq 13
$$

Theorem 3.21 states that $\forall n \in \mathbb{N} \mid n \geq 13$, the sum of all error terms made at every step of estimation of $\pi_{(n)}$ diverges. In particular, it states that:

$$
\begin{aligned}
\left(T L i_{(n)}-\pi_{(n)}\right)=\left(\sum_{p_{(i+1)}=5}^{p_{(n)}}\left\{\left(\int_{\theta_{1}}^{\theta_{2}} \frac{d t}{\log t}\right)-1\right\}\right. & \left.+\left(\int_{2}^{\theta_{3}} \frac{d t}{\log t}-\pi_{3}\right)\right) \\
& >\sum_{p \leq n} \frac{1}{p} \quad \forall n \in \mathbb{N} \mid n \geq 983
\end{aligned}
$$

Where $\quad\left(\int_{2}^{\theta_{3}} \frac{d t}{\log t}-\pi_{3}\right)=\left(\int_{2}^{\log 6} \frac{d t}{\log t}-2\right) \approx-2.3266013098834977$

On the basis of Theorem 3.18 define $\forall n \in \mathbb{N} \mid n \geq 983$ the estimation error:

Definition 2.1 (Estimation error over $\mathcal{G} s_{(n)}$ ).

$$
\mathcal{E}_{(t)}=\left(T L i_{(t)}-T L i_{(n)}\right)-\left(\pi_{(t)}-\pi_{(n)}\right)=\sum_{n<p_{i+1} \leq t}\left[\left(\int_{\theta_{1}}^{\theta_{2}} \frac{d t}{\log t}\right)-1\right]
$$

and the residual estimation error: 
Definition 2.2 (Residual error over $\mathcal{G}_{\left.s_{(n)}\right)}$.

$$
\mathcal{R} \mathcal{E}_{(t)}=T L i_{(t)}-T L i_{(n)}-\left(\pi_{(t)}-\pi_{(n)}\right)-\sum_{n<p \leq t} \frac{1}{p} \quad \forall n \in \mathbb{N} \mid n \geq 983
$$

By Theorem 3.21 therefore:

$$
\mathcal{R E}_{(t)}>0 \quad \forall n \in \mathbb{N} \mid n \geq 983
$$

Thus, the estimation error over the interval $\mathcal{G} s_{(n)}$ equals:

$$
\mathcal{E E}_{(t)}=\sum_{n<p \leq t} \frac{1}{p}+\mathcal{R E}_{(t)} \quad \forall n \in \mathbb{N} \mid n \geq 983
$$

Due to the finite and very short length of the interval $\mathcal{G} s_{(n)}$ the limit is:

$$
\lim _{n \rightarrow \infty}\left(\sum_{n<p_{i+1} \leq t}\left[\left(\int_{\theta_{1}}^{\theta_{2}} \frac{d t}{\log t}\right)-1\right]\right)=\lim _{n \rightarrow \infty}\left(\sum_{n<p \leq t} \frac{1}{p}\right) \rightarrow 0
$$

Equation 2.5 implies that the largest estimation error $\mathcal{E E}_{(t)}$ occurs for low $n \in \mathbb{N}$.

\subsection{The Maximum bound for the number of primes in $\mathcal{G} s_{(n)}$.}

This section establishes the Maximum bound for primes within the interval $\mathcal{G} s_{(n)}$. The Infimum for primes in the interval $\mathcal{G}_{s_{(n)}}$ is given by Theorem 3.2. The average number of primes within the interval is given by the PNT:

$$
\frac{\mathcal{G} s_{(n)}}{\log (t)}=\frac{5\left(\log _{10} n\right)^{2}-\frac{15}{8}\left(\log _{10} n\right)}{\log \left(n+5\left(\log _{10} n\right)^{2}-\frac{15}{8}\left(\log _{10} n\right)\right)} \quad \forall n \in \mathbb{N} \mid n \geq 11
$$

Montgomery and Vaughan [26] in 1973, using the Large Sieve proved that:

$$
\left(\pi_{(x)}-\pi_{(x-y)}\right) \leq \frac{2 y}{\log y} \quad \forall x, y \in \mathbb{N} \mid 1<y \leq x
$$

Paul Erdös [10] in his 1980 paper, raised the problem of the number of primes within a short interval, and he conjectured that the number of primes within a short interval may be estimated by using:

Conjecture 2.3 (Primes In Short Intervals - Paul Erdös).

$$
\pi_{x}-\pi_{y} \leq \frac{b(x-y)}{\log (x)} \quad \text { for every } y \leq x-(\log (x))^{b}
$$

Where b is sufficiently large absolute constant.

Substituting into the inequality $2.8 ; y=n, x=n+c, b=2$ we obtain:

$$
\pi_{(n+c)}-\pi_{(n)} \leq \frac{2 c}{(\log t)}=\frac{2 \times\left(5\left(\log _{10} n\right)^{2}-\frac{15}{8}\left(\log _{10} n\right)\right)}{\log \left(n+\left(5\left(\log _{10} n\right)^{2}-\frac{15}{8}\left(\log _{10} n\right)\right)\right)}
$$

Inequality 2.9 , clearly implements the symmetry argument based on the PNT (equation 2.6). We assert, that the Maximum for primes within the interval $c=\mathcal{G} s_{(n)}$ is given by Theorem 2.4 . 
Theorem 2.4 (Maximum For Primes In $\mathcal{G} s_{(n)}$ ). by:

The maximum number of primes within the interval $\mathcal{G} s_{(n)}$ from $n$ to $t$ is given

$$
\begin{array}{r}
\mathcal{M} \mathcal{A} \mathcal{X}_{(t)}=\frac{2 \mathcal{G} s_{(n)}}{\log (t)}=\frac{2 \times\left(5\left(\log _{10} n\right)^{2}-\frac{15}{8}\left(\log _{10} n\right)\right)}{\log \left(n+\left(5\left(\log _{10} n\right)^{2}-\frac{15}{8}\left(\log _{10} n\right)\right)\right)}>\left(\pi_{(t)}-\pi_{(n)}\right) \\
\forall n \in \mathbb{N} \mid n \geq 11
\end{array}
$$

Proof.

$$
\mathcal{M} \mathcal{A} \mathcal{X}_{(t)}=\frac{2 \times\left(5\left(\log _{10} n\right)^{2}-\frac{15}{8}\left(\log _{10} n\right)\right)}{\log \left(n+\left(5\left(\log _{10} n\right)^{2}-\frac{15}{8}\left(\log _{10} n\right)\right)\right)} \lesssim \frac{10}{\log 10}\left(\left(\log _{10} n\right)-\frac{3}{8}\right)
$$

In fact, the difference of terms of 2.11 i.e. LHS less RHS, at $n=11$ attains approximately -0.296956805 . The limit of the difference:

$$
\lim _{n \rightarrow \infty}\left[\frac{2 \times\left(5\left(\log _{10} n\right)^{2}-\frac{15}{8}\left(\log _{10} n\right)\right)}{\log \left(n+\left(5\left(\log _{10} n\right)^{2}-\frac{15}{8}\left(\log _{10} n\right)\right)\right)}-\frac{10}{\log 10}\left(\left(\log _{10} n\right)-\frac{3}{8}\right)\right] \rightarrow 0
$$

tends asymptotically towards zero. Thus obviously, $\mathcal{M} \mathcal{A} \mathcal{X}_{(t)}$ diverges as n increases unboundedly. The tailored integral $T L i_{(n)}$ and the sum $\sum_{n<p \leq t} \frac{1}{p}>0$ are weakly monotone, increasing functions. From the definition 2.2 we have:

$$
T L i_{(t)}-T L i_{(n)}-\sum_{n<p \leq t} \frac{1}{p}=\left(\pi_{(t)}-\pi_{(n)}\right)+\mathcal{R} \mathcal{E}_{(t)} \quad \forall n \in \mathbb{N} \mid n \geq 983
$$

Suppose that Theorem 2.4 for $n \in \mathbb{N} \mid n \geq 983$ is false. This implies that,

$$
\frac{2 \times\left(5\left(\log _{10} n\right)^{2}-\frac{15}{8}\left(\log _{10} n\right)\right)}{\log \left(n+\left(5\left(\log _{10} n\right)^{2}-\frac{15}{8}\left(\log _{10} n\right)\right)\right)}<\left(\pi_{(t)}-\pi_{(n)}\right)
$$

Clearly,

$$
\left(\pi_{(t)}-\pi_{(n)}\right)<\left(\pi_{(t)}-\pi_{(n)}\right)+\mathcal{R} \mathcal{E}_{(t)}=T L i_{(t)}-T L i_{(n)}-\sum_{n<p \leq t} \frac{1}{p}
$$

Hence, substituting the RHS of 2.13 into 2.12, we obtain in accordance with the hypothesis:

$$
\frac{2 \times\left(5\left(\log _{10} n\right)^{2}-\frac{15}{8}\left(\log _{10} n\right)\right)}{\log \left(n+\left(5\left(\log _{10} n\right)^{2}-\frac{15}{8}\left(\log _{10} n\right)\right)\right)}+T L i_{(n)}+\sum_{n<p \leq t} \frac{1}{p}-T L i_{(t)}<0
$$


However, at $n=983$ the inequality 2.14 attains $\sim 18.696357205817502$ and increases as $n$ increases unboundedly. Since inequality 2.14 generates a positive sequence of values, we implement the Cauchy Root Test:

$$
\begin{aligned}
\lim _{n \rightarrow \infty} \sqrt[\left(\pi_{n}\right)]{a_{n}}= & \sqrt[\left(\pi_{n}\right)]{\left(\frac{2 \times\left(5\left(\log _{10} n\right)^{2}-\frac{15}{8}\left(\log _{10} n\right)\right)}{\log \left(n+\left(5\left(\log _{10} n\right)^{2}-\frac{15}{8}\left(\log _{10} n\right)\right)\right)}+T L i_{(n)}+\sum_{n<p \leq t} \frac{1}{p}-T L i_{(t)}\right)}
\end{aligned}
$$

The Root Test, at $n=22$ attains $\sim 1.1736950736631349$, at $n=983$ the test already descended to $\sim 1.017797047552908$ and continues to converge asymptotically, strictly from above to 1 . Consequently, by the definition of the Cauchy's Root Test, the series formed from the terms of the sequence 2.14 diverges. Therefore, we have a contradiction to the initial hypothesis. Hence, $\forall n \in \mathbb{N} \mid n \geq 983$ the inequality is valid:

$$
\frac{2 \times\left(5\left(\log _{10} n\right)^{2}-\frac{15}{8}\left(\log _{10} n\right)\right)}{\log \left(n+\left(5\left(\log _{10} n\right)^{2}-\frac{15}{8}\left(\log _{10} n\right)\right)\right)}>T L i_{(t)}-T L i_{(n)}-\sum_{n<p \leq t} \frac{1}{p}
$$

Consequently, for all $n \in \mathbb{N} \mid n \geq 983$ Theorem 2.4 holds. For all $n \in \mathbb{N} \mid 11 \leq$ $n \leq 983$, direct evaluation evidently confirms that inequality 2.14 holds in this range as well. Please refer to Figure 2. Hence, Theorem 2.4 holds $\forall n \in \mathbb{N} \mid n \geq 11$.
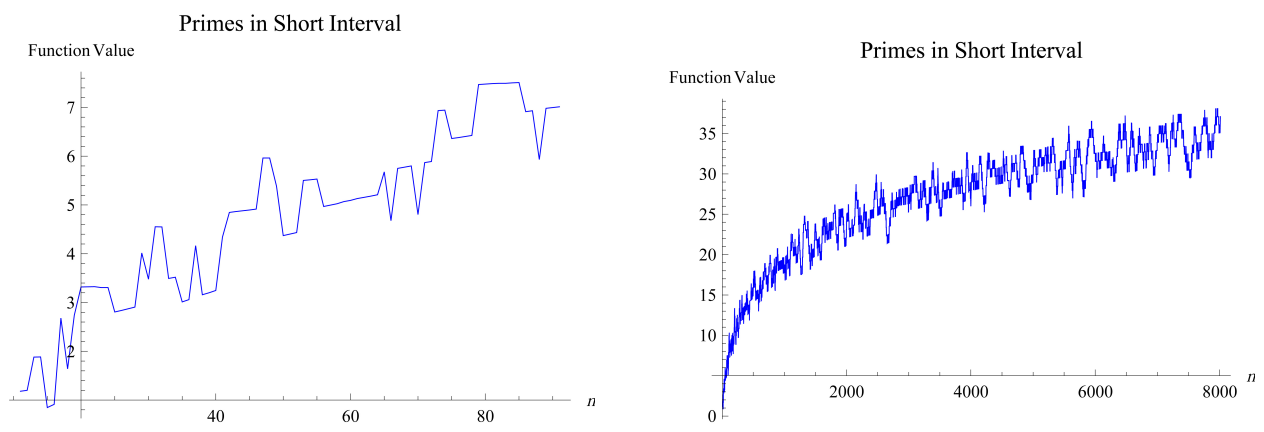

FiguRE 2. shows the graphs of inequality 2.14 within the interval at every $n \in \mathbb{N} \mid 11 \leq n \leq 91$ in the left figure, and $11 \leq n \leq 8000$ in the right figure. 
Corollary 2.5 (Summary Of The Distribution Of Primes In $\mathcal{G} s_{(n)}$ ).

On the basis of Theorem 3.2 and Theorem 2.4 the density of prime numbers within the interval $\mathcal{G} s_{(n)}$, from $\mathrm{n}$ to $\left(n+\mathcal{G} s_{(n)}\right)$ for all $n \in \mathbb{N} \mid n \geq 11$ has the following bounds:

$$
\begin{aligned}
& 1 \leq \pi_{(t)}-\pi_{(n)}<\frac{2 \mathcal{G} s_{(n)}}{\log (t)}=\frac{2 \times\left(5\left(\log _{10} n\right)^{2}-\frac{15}{8}\left(\log _{10} n\right)\right)}{\log \left(n+\left(5\left(\log _{10} n\right)^{2}-\frac{15}{8}\left(\log _{10} n\right)\right)\right)} \\
& \text { with the average: } \frac{\mathcal{G} s_{(n)}}{\log (t)}=\frac{\left(5\left(\log _{10} n\right)^{2}-\frac{15}{8}\left(\log _{10} n\right)\right)}{\log \left(n+\left(5\left(\log _{10} n\right)^{2}-\frac{15}{8}\left(\log _{10} n\right)\right)\right)}
\end{aligned}
$$

\subsection{Historical reflection. Density of primes in a short interval.}

Atle Selberg [35] researched the problem of the density of primes in small interval:

$$
\left\{n, n+\Phi_{n}\right\}
$$

where $\Phi_{n}$ is positive, increasing function and for $n>0$ :

$$
\lim _{n \rightarrow \infty}\left(\Phi_{n}\right) \rightarrow \infty
$$

including:

$$
\lim _{n \rightarrow \infty}\left(\frac{\Phi_{n}}{n}\right) \rightarrow 0
$$

Assuming Riemann's hypothesis Selberg was able to prove that:

$$
\pi_{\left(n+\Phi_{n}\right)}-\pi_{(n)} \sim \frac{\Phi_{n}}{\log (n)} \quad \text { where } \Phi_{n}>(\log (n))^{(2+\epsilon)}
$$

Over the years questions were posed as to the nature of the function $\Phi_{n}$. In 1985 H. Maier [25] proved that:

$$
\limsup _{n \rightarrow \infty}\left(\frac{\pi_{\left(n+\Phi_{n}\right)}-\pi_{(n)}}{\Phi_{n} / \log (n)}\right)>1 \text { for } \Phi_{n}=(\log (n))^{\lambda} \text { s.t. } \lambda>1
$$

as well as:

$$
\liminf _{n \rightarrow \infty}\left(\frac{\pi_{\left(n+\Phi_{n}\right)}-\pi_{(n)}}{\Phi_{n} / \log (n)}\right)<1
$$

The theory presented thus far, makes it possible to solve these problems on the interval $\mathcal{G}_{s_{(n)}}$. Theorem 3.2 gives the maximum gaps standard. Clearly, $\mathcal{G} s_{(n)}$ is a positive, increasing function s.t.

$$
\lim _{n \rightarrow \infty}\left(\mathcal{G} s_{(n)}\right) \rightarrow \infty
$$

and

$$
\lim _{n \rightarrow \infty}\left(\frac{\mathcal{G} s_{(n)}}{n}\right) \rightarrow 0
$$


From the Corollary 2.5 which specifies the distribution of primes in the interval $\mathcal{G} s_{(n)}$ for all $n \in \mathbb{N} \mid n \geq 11$ we substitute therefore into 2.18 obtaining:

$$
\begin{array}{r}
\limsup _{n \rightarrow \infty}\left(\frac{2 \times\left(5\left(\log _{10} n\right)^{2}-\frac{15}{8}\left(\log _{10} n\right)\right)}{\log (t)} \times \frac{\log (n)}{\left(5\left(\log _{10} n\right)^{2}-\frac{15}{8}\left(\log _{10} n\right)\right)}\right) \\
=\limsup _{n \rightarrow \infty}\left(\frac{2 \log (n)}{\log (t)}\right)=2
\end{array}
$$

Similarly substituting into 2.19 we obtain:

$$
\liminf _{n \rightarrow \infty}\left(\frac{1}{\frac{5\left(\log _{10} n\right)^{2}-\frac{15}{8}\left(\log _{10} n\right)}{\log (n)}}\right)=\liminf _{n \rightarrow \infty}\left(\frac{\log (10)}{5\left(\log _{10} n\right)-\frac{15}{8}}\right)=0
$$

Clearly corroborating Maier's results. For graphical presentation of Equations 2.20 and 2.21 please refer to Fig. 3. The relation 2.17 then becomes:

$$
\pi_{\left(n+\mathcal{G} s_{(n)}\right)}-\pi_{(n)} \sim \frac{\mathcal{G} s_{(n)}}{\log (n)}=\frac{5}{\log (10)}\left(\left(\log _{10} n\right)-\frac{3}{8}\right)
$$

Which leads to the next section's problem: the Second Hardy and Littlewood's conjecture.
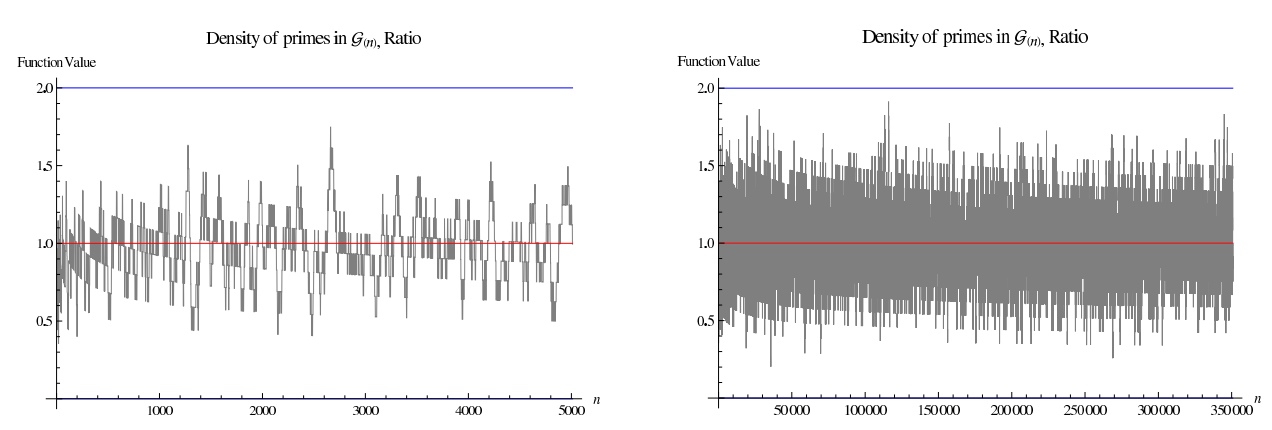

Figure 3. The drawings show the graphs of the ratio of the actual prime counts $\left(\pi_{\left(n+\mathcal{G}_{(n)}\right)}-\pi_{(n)}\right) /\left(\mathcal{G}_{(n)} / \log (n)\right)$ (grey). The figures are drawn at every $n \in \mathbb{N} \mid n \geq 8$ within the range.

\subsection{The Second Hardy and Littlewood's conjecture.}

In 1923 Godfrey H. Hardy and John E. Littlewood postulated a conjecture on the basis of a limited empirical evidence:

$$
\pi_{k}>\pi_{(n+k)}-\pi_{n}
$$

Although some believe that the conjecture may be incorrect, yet no one has actually proven it to be true or false. In this section a proof is presented which establishes the truth of the conjecture 2.22 on the interval $\mathcal{G} s_{(n)}$. Theorems: 3.2 and 2.4 make it possible to verify and prove the conjecture. 
Theorem 2.6 (Hardy and Littlewood Conjecture).

The conjecture postulated by G. Hardy and J. Littlewood is valid on the interval $\mathcal{G} s_{(n)}$ given by Theorem 3.2:

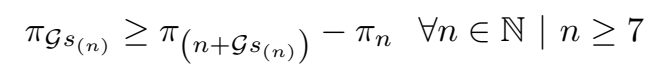

Proof.

By PNT the number of primes within the interval fixed at 1 and terminating at $\mathcal{G} s_{(n)}$ is asymptotic to:

$$
\pi_{\left(\mathcal{G} s_{(n)}\right)} \sim \frac{\mathcal{G} s_{(n)}}{\log \left(\mathcal{G} s_{(n)}\right)}
$$

Rosser and Schoenfeld [32] in 1962, proved for all $n \mid n>10$ that:

$$
\pi_{(n)}>\frac{n}{\log n}
$$

The Maximum for the number of primes within the interval $\mathcal{G} s_{(n)}$ from $n$ to $t$ is given by Theorem 2.4:

$$
\pi_{\left(n+\mathcal{G} s_{(n)}\right)}-\pi_{n} \leq \frac{2 \mathcal{G} s_{(n)}}{\log (t)}=\frac{2 \times\left(5\left(\log _{10} n\right)^{2}-\frac{15}{8}\left(\log _{10} n\right)\right)}{\log \left(n+\left(5\left(\log _{10} n\right)^{2}-\frac{15}{8}\left(\log _{10} n\right)\right)\right)}
$$

The ratio of the two estimates is given by:

$$
\frac{\pi_{\left(\mathcal{G} s_{(n)}\right)}}{\pi_{\left(n+\mathcal{G}_{(n)}\right)}-\pi_{n}} \geq \frac{\log (t)}{2 \log \left(\mathcal{G} s_{(n)}\right)}=\frac{\log \left(n+\left(5\left(\log _{10} n\right)^{2}-\frac{15}{8}\left(\log _{10} n\right)\right)\right)}{2 \log \left(5\left(\log _{10} n\right)^{2}-\frac{15}{8}\left(\log _{10} n\right)\right)}
$$

Thus, for all $n \in \mathbb{N} \mid n \geq 401$, the limit of the ratio 2.24 is given by:

$$
\begin{gathered}
\lim _{n \rightarrow \infty}\left(\frac{\log \left(n+\left(5\left(\log _{10} n\right)^{2}-\frac{15}{8}\left(\log _{10} n\right)\right)\right)}{2 \log \left(5\left(\log _{10} n\right)^{2}-\frac{15}{8}\left(\log _{10} n\right)\right)}\right) \\
=\lim _{n \rightarrow \infty}\left(\frac{\left(\log (n)+\log \left(1+\frac{\left(5\left(\log _{10} n\right)^{2}-\frac{15}{8}\left(\log _{10} n\right)\right)}{n}\right)\right)}{2 \log \left(5\left(\log _{10} n\right)^{2}-\frac{15}{8}\left(\log _{10} n\right)\right)}\right)
\end{gathered}
$$

Clearly,

$$
\lim _{n \rightarrow \infty}\left(\frac{\log (n)}{2 \log \left(5\left(\log _{10} n\right)^{2}-\frac{15}{8}\left(\log _{10} n\right)\right)}\right) \rightarrow \infty
$$

while,

$$
\lim _{n \rightarrow \infty}\left(\frac{\log \left(1+\frac{\left(5\left(\log _{10} n\right)^{2}-\frac{15}{8}\left(\log _{10} n\right)\right)}{n}\right)}{2 \log \left(5\left(\log _{10} n\right)^{2}-\frac{15}{8}\left(\log _{10} n\right)\right)}\right) \rightarrow 0
$$


Which consequently establishes the divergence of the limit of the ratio 2.24 :

$$
\begin{gathered}
\lim _{n \rightarrow \infty}\left(\frac{\log \left(n+\left(5\left(\log _{10} n\right)^{2}-\frac{15}{8}\left(\log _{10} n\right)\right)\right)}{2 \log \left(5\left(\log _{10} n\right)^{2}-\frac{15}{8}\left(\log _{10} n\right)\right)}\right) \\
=\lim _{n \rightarrow \infty}\left(\frac{\left(\log (n)+\log \left(1+\frac{\left(5\left(\log _{10} n\right)^{2}-\frac{15}{8}\left(\log _{10} n\right)\right)}{n}\right)\right)}{2 \log \left(5\left(\log _{10} n\right)^{2}-\frac{15}{8}\left(\log _{10} n\right)\right)}\right) \rightarrow \infty
\end{gathered}
$$

At $n=401$ the ratio 2.24 attains the value $\approx 3.33333$ and increases as $n$ increases unboundedly. This implies that Theorem 2.6 holds for all $n \in \mathbb{N} \mid n \geq 401$. For all $n \in \mathbb{N} \mid 7<n \leq 401$ direct computation verifies that the actual counts $\pi_{\left(n+\mathcal{G s}_{(n)}\right)}-$ $\pi_{n} \leq \pi_{\left(\mathcal{G}_{(n)}\right)}$, consequently Theorem 2.6 holds in this range. Please refer to Fig. 4. Therefore Theorem 2.6 holds $\forall n \in \mathbb{N} \mid n \geq 7$. This concludes the proof of Theorem 2.6.

Inequality 2.23 compares the number of primes contained within two intervals of equal length which are some distance apart. The function $\pi_{\mathcal{G} s_{(n)}}$ counts the number of primes within the interval beginning at 1 and terminating at $\mathcal{G} s_{(n)}$. At the same time $\pi_{\left(n+\mathcal{G} s_{(n)}\right)}-\pi_{n}$ counts the number of primes within the interval of precisely the same length, yet beginning at $n$ and terminating at $t=n+\mathcal{G} s_{(n)}$.
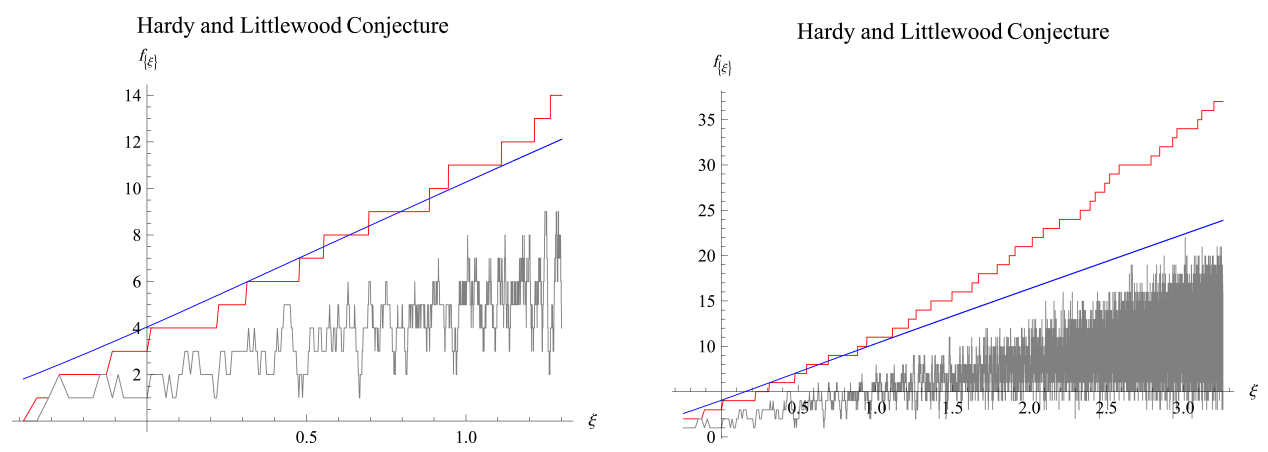

Figure 4 . The drawings show the graphs of $\pi_{\mathcal{G} s_{(n)}}$ (Red), the Maximum for primes within the interval $\mathcal{G} s_{(n)}$ (Blue) and the difference $\pi_{\left(n+\mathcal{G}_{(n)}\right)}-\pi_{n}$ (Grey). The figure shows the last intersection of the Maximum with $\pi_{\mathcal{G}_{(n)}}$ at $\xi \approx 0.886048$, which corresponds to $x \in \mathbb{R} \mid x \approx 400$. $\pi_{\mathcal{G}_{(n)}}$ exceeds the Maximum from $n=401$ onwards. The L.H. figure is drawn w.r.t. $\xi$, at all $n \in \mathbb{N} \mid 7 \leq n \leq 1500$; while the R.H. figure is drawn at all $n \in \mathbb{N} \mid 11 \leq n \leq 750000$.

Remark 2.1. Although the extent of numerical verification possible on PC remains limited, (Mathematica ${ }^{\circledR}$ will calculate the number of primes for any given $\mathrm{n}$ up to $\left.n \leq 2 \times 10^{14}\right)$; since the value of $2 \times 10^{14}$ is the length of the interval $k$ in 2.22 , this 
implies that for all practical reasons we may numerically verify the conjecture at any $n \in \mathbb{N}$ such that:

$$
10 \leq n \leq 3.219858313513683 \times 10^{6324555}
$$

\subsection{The Merit of a Prime Gap and Harald Cramér's Conjecture.}

The merit of a prime gap is defined as the ratio of the size of a gap beginning at $p_{n}$ and $\log \left(p_{n}\right)$. It indicates the relative size of a given gap as compared to the average prime gap about $p_{n}$ as given by the PNT.

Theorem 2.7 (Prime Gap Merit).

The maximal prime gap merit diverges as $p_{n} \in \mathbb{N}$ increases unboundedly:

$$
\lim _{p_{n} \rightarrow \infty}\left(\mathfrak{M}_{p_{n}}=\frac{\left(p_{n+1}-p_{n}\right)}{\log \left(p_{n}\right)}\right) \rightarrow \infty
$$

Proof.

Theorem 3.2 gives us the Supremum for the maximal prime gaps, this means that for all $p_{n} \in \mathbb{N} \mid p_{n} \geq 11$, we have:

$$
\mathfrak{M}_{p_{n}}=\frac{\left(p_{n+1}-p_{n}\right)}{\log \left(p_{n}\right)} \leq \frac{\left(5\left(\log _{10} p_{n}\right)^{2}-\frac{15}{8}\left(\log _{10} p_{n}\right)\right)}{\log \left(p_{n}\right)}=\frac{5}{\log (10)}\left(\left(\log _{10} p_{n}\right)-\frac{3}{8}\right)
$$

Consequently, the Supremum on the prime gap merit is given by:

$$
\operatorname{Sup}\left(\mathfrak{M}_{p_{n}}\right)=\frac{5}{\log (10)}\left(\left(\log _{10} p_{n}\right)-\frac{3}{8}\right)=\frac{5}{(\log 10)^{2}}\left(\log p_{n}-\frac{3 \log 10}{8}\right)
$$

Clearly,

$$
\lim _{p_{n} \rightarrow \infty}\left(\operatorname{Sup}\left(\mathfrak{M}_{p_{n}}\right)\right)=\lim _{p_{n} \rightarrow \infty}\left(\frac{5}{(\log 10)^{2}}\left(\log p_{n}-\frac{3 \log 10}{8}\right)\right) \rightarrow \infty
$$

Now, Theorem 3.5 gives us the Lower Bound for the maximal prime gaps. This means, that for all $p_{n} \in \mathbb{N} \mid p_{n} \geq 7$, in the case of maximal gaps the Lower Bound on the prime gap merit is given by:

$$
L B\left(\mathfrak{M}_{p_{n}}\right)=\left\lfloor\frac{\mathcal{G} s_{(n)}}{2 \log \left(p_{n}\right)}\right\rfloor \leq \frac{p_{(n+1)}-p_{(n)}}{\log \left(p_{n}\right)}=\mathfrak{M}_{p_{n}}
$$

Hence,

$$
\begin{aligned}
L B\left(\mathfrak{M}_{p_{n}}\right)=\left\lfloor\frac{\left(5\left(\log _{10} p_{n}\right)^{2}-\frac{15}{8}\left(\log _{10} p_{n}\right)\right)}{2 \log \left(p_{n}\right)}\right\rfloor \\
=\left\lfloor\frac{5}{2(\log 10)^{2}}\left(\log p_{n}-\frac{3 \log 10}{8}\right)\right\rfloor \leq \mathfrak{M}_{p_{n}}
\end{aligned}
$$

Clearly,

$$
\lim _{p_{n} \rightarrow \infty}\left(L B\left(\mathfrak{M}_{p_{n}}\right)\right)=\lim _{p_{n} \rightarrow \infty}\left(\left\lfloor\frac{5}{2(\log 10)^{2}}\left(\log p_{n}-\frac{3 \log 10}{8}\right)\right\rfloor\right) \rightarrow \infty
$$


Hence, the bounds on the maximal prime gaps merit $\mathfrak{M}_{p_{n}}$ are:

$$
\begin{aligned}
\left\lfloor\frac{5}{2(\log 10)^{2}}\left(\log p_{n}-\frac{3 \log 10}{8}\right)\right\rfloor \leq & \frac{p_{(n+1)}-p_{(n)}}{\log \left(p_{n}\right)}=\mathfrak{M}_{p_{n}} \\
& \leq \frac{5}{(\log 10)^{2}}\left(\log p_{n}-\frac{3 \log 10}{8}\right)
\end{aligned}
$$

Consequently, since $\mathfrak{M}_{p_{n}}$ is 'sandwiched' between $L B\left(\mathfrak{M}_{p_{n}}\right)$ and $\operatorname{Sup}\left(\mathfrak{M}_{p_{n}}\right)$, from 2.29 and 2.32 we obtain:

$$
\begin{array}{r}
\lim _{p_{n} \rightarrow \infty}\left(\left\lfloor\frac{5}{2(\log 10)^{2}}\left(\log p_{n}-\frac{3 \log 10}{8}\right)\right\rfloor\right)=\lim _{p_{n} \rightarrow \infty}\left(\mathfrak{M}_{p_{n}}\right) \\
=\lim _{p_{n} \rightarrow \infty}\left(\frac{5}{(\log 10)^{2}}\left(\log p_{n}-\frac{3 \log 10}{8}\right)\right) \rightarrow \infty
\end{array}
$$

Thus, Theorem 2.7 holds as stated; Concluding the proof.

Now, Lemma 3.6 implies that:

$$
\sum_{p \leq n} \log p=\log p \sharp<p \leq n \quad \forall n \in \mathbb{N} \mid n \geq 3
$$

Further, for all $n \in \mathbb{N} \mid n \geq 3$ we have that:

$$
\sum_{p \leq n}(\log p)^{2}<\left(\log p_{n}\right) \sum_{p \leq n} \log p
$$

Consequently we have that:

$$
\left(\log p_{n}-1\right) \sum_{p \leq n} \log p=\left(\log p_{n}-1\right)\left(\log p_{n} \sharp\right)<\left(\log p_{n}-1\right)\left(p_{n}\right)
$$

By Theorem 3.9 and Lemmas 3.10 through 3.12 we have that for all $p_{n} \in \mathbb{N} \mid p_{n} \geq$ 11:

$$
\begin{gathered}
L B_{p_{n}}=\left(\log p_{n}-1\right)\left(p_{n}-2 \sqrt{p_{n}}\right)<\operatorname{Inf}=\left[\left(\left(\log p_{n}-1\right) \log p_{n} \sharp\right)+\log 2\right] \\
<\sum_{p \leq n}(\log p)^{2}<\left[\left(\left(p_{n}^{(-G M)}+1\right)\left(\log p_{n}-1\right) \log p_{n} \sharp\right)+\frac{\pi}{2}\right]=\text { Sup } \\
<\left(\log p_{n}-1\right) p_{n}+1=U B_{p_{n}}
\end{gathered}
$$

where $p_{n}$ is the biggest prime $p \leq n$. H. Cramér in his paper [4] showed that for any $\epsilon>0$ the relation:

$$
\sum_{p_{n} \leq x}\left(\frac{\left(p_{n+1}-p_{n}\right)}{\log \left(p_{n}\right)}\right)^{2}=\mathcal{O}\left(x(\log x)^{(1+\epsilon)}\right)
$$

conditionally holds providing that the Riemann's Hypothesis is true. This section provides an unconditional proof of the relation 2.36. 
Theorem 2.8 (Sum Of Squares Of $\mathfrak{M}_{p_{n}}$ Terms).

For $m, n \in \mathbb{N} \mid n \geq 13$ and $p_{n}$ the largest prime $p \leq n$, the relation holds:

$$
\begin{aligned}
\sum_{p_{m} \leq n}\left(\frac{\left(p_{m+1}-p_{m}\right)}{\log \left(p_{m}\right)}\right)^{2} & <\left(\frac{5}{(\log 10)^{2}}\right)^{2}\left(\log p_{n}\right) \log p_{n} \sharp \\
< & \left(\frac{5}{(\log 10)^{2}}\right)^{2}\left(\log p_{n}\right) p_{n}<\left(\log p_{n}\right) p_{n} \leq(\log n) n
\end{aligned}
$$

Proof.

For $p_{m} \in \mathbb{N} \mid p_{m} \geq 13$ by Theorem 3.1 and Lemma 2.7 we have:

$$
\frac{p_{(m+1)}-p_{m}}{\log p_{m}} \leq \frac{5}{(\log 10)^{2}}\left(\log p_{m}-\frac{3 \log 10}{8}\right)<\frac{5}{(\log 10)^{2}}\left(\log p_{m}\right)
$$

Therefore, from inequalities $2.27,2.35$ and 2.38 for all $n \in \mathbb{N} \mid n \geq 13$

$$
\begin{aligned}
& \sum_{p_{m} \leq n}\left(\frac{p_{(m+1)}-p_{m}}{\log p_{m}}\right)^{2} \leq\left(\frac{5}{(\log 10)^{2}}\right)^{2} \sum_{p_{m} \leq n}\left(\log p_{m}-\frac{3 \log 10}{8}\right)^{2} \\
&<\left(\frac{5}{(\log 10)^{2}}\right)^{2} \sum_{p_{m} \leq n}\left(\log p_{m}\right)^{2}<\left(\frac{5}{(\log 10)^{2}}\right)^{2}\left(\log p_{n}\right) \log p_{n} \sharp \\
&<\left(\frac{5}{(\log 10)^{2}}\right)^{2}\left(\log p_{n}\right) p_{n}<\left(\log p_{n}\right) p_{n} \leq(\log n) n
\end{aligned}
$$

At $p=13$ :

$$
\begin{aligned}
& \sum_{p_{m} \leq n}\left(\frac{p_{(m+1)}-p_{m}}{\log p_{m}}\right)^{2}=14.292860751997972 \\
& \quad<\left(\frac{5}{(\log 10)^{2}}\right)^{2} \sum_{p_{m} \leq n}\left(\log p_{m}-\frac{3 \log 10}{8}\right)^{2}=15.56015603961242
\end{aligned}
$$

Therefore, Theorem 2.8 holds, simultaneously proving the relation 2.36 hence, concluding the proof.

\section{REFERENCES}

[1] R.J. Backlund, Über die Differenzen zwischen den Zahlen, die zu den ersten $n$ Primzahlen teilerfremd sind. Commentationes in honorem Ernesti Leonardi Lindelöf, Annales Acad. Sci. Fenn. 32 (1929), no. 2, 1-9.

[2] A. Brauer and H. Zeitz, Über eine zahlentheoretische Behauptung von Legendre, Sitzungsbericht, Berliner Math. Ges. 29 (1930), 116-125.

[3] Chris K. Caldwell, The gaps between primes, 2006. http://primes.utm.edu/notes/gaps.html.

[4] Harald Cramer, On the order of magnitude of the difference between consecutive prime numbers, Acta Arithmetica (1936).

[5] R. Crandall and C. Pomerance, Prime numbers, a computational perspective, Springer Verlag, New York, 2005.

[6] P. Dusart, Autour de la fonction qui compte le nombre de nombres premiers, Limoges, 1998.

[7] Paul Erdös, On the difference of consecutive primes, Quarterly Journal Of Mathematics (1935). 

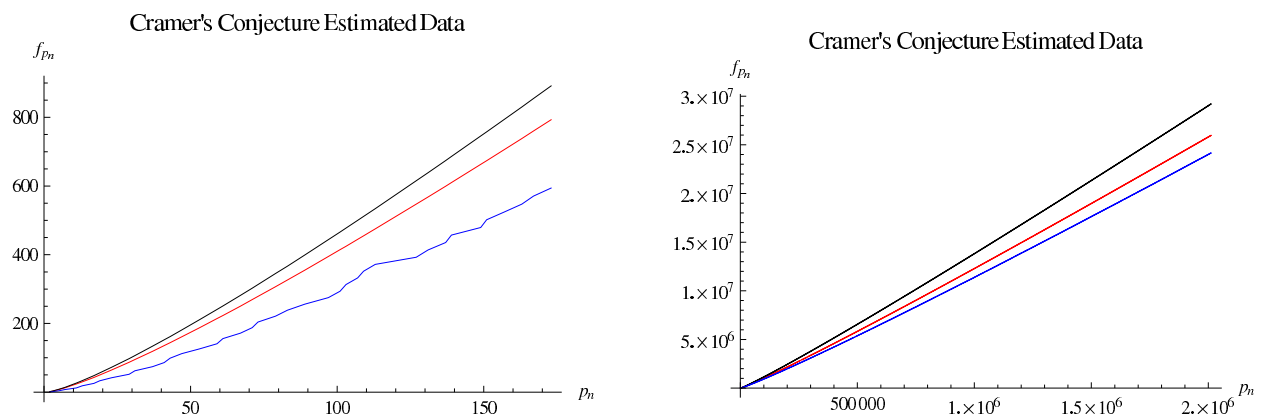

Figure 5. The graphs show the drawing of $n(\log n)$ (black), the drawing of the estimates $\left(5^{2} /(\log 10)^{4}\right)(n(\log n))$ (red) and the graph of the estimates of the sum 2.37 by $\left(5^{2} /(\log 10)^{4}\right) \sum_{p \leq n}\left(\log p_{n}\right)^{2}$ (blue). The figures are drawn with respect to $p_{n} \in \mathbb{N}$.

[8] _ Some unsolved problems, Publications Of The Mathematical Institute Of The Hungarian Academy Of Sciences (1961).

[9] _ A survey of problems in combinatorial number theory, Annals Of Discrete Mathematics (1980).

[10] Paul Erdös and E.G. Strauss, Remarks on the differences between consecutive primes, Elem. Math. 35 (1980), 115-118.

[11] Jan Feliksiak, The elementary proof of the Riemann's Hypothesis, MDPI, AG, https://doi.org/10.20944/preprints202006.0365.v2 (2020).

[12] AG, https://doi.org/10.20944/preprints202006.0366.v1 (2020).

[13] _ The Brocard's Conjecture, ScienceOpen, Inc, https://doi.org/10.14293/S21991006.1.SOR-.PPIH4LV.v1 (2021).

[14] _ Maximal prime gaps bounds, ScienceOpen, https://doi.org/10.14293/S21991006.1.SOR-.PPWVKRR.v1 (2021).

[15] _ The Nicholson's Conjecture, ScienceOpen, Inc, https://doi.org/10.14293/S21991006.1.SOR-.PPT1JIB.v1 (2021).

[16] _ The Binary Goldbach Conjecture, ScienceOpen, Inc, https://doi.org/10.14293/S2199-1006.1.SOR-.PPIU46T.v1 (2021).

$[17]$, Bounds on the sum of log $p$ terms squared, MDPI, AG, https://doi.org/10.20944/preprints202107.0221.v1 (2021).

[18] P. Gallagher, On the distribution of primes in short intervals, Mathem. 23 (1976), 4-9.

[19] Andrew Granville, Harald Cramer and the distribution of prime numbers, Scandinavian Actuarial Journal (1995).

[20] G.H. Hardy and E.M. Wright, An introduction to the theory of numbers, Oxford University Press, London, 1968.

[21] M. Hassani, A refinement of Mandl's inequality and approximation of the product $p_{1} p_{2} \cdots p_{n}$, arXiv:math/0606765v1[math.NT] (2006).

[22] D.R. Heath-Brown, Differences between consecutive primes, Jahresber. Deutsch. Mathem. Ver. 90 (1988), 71-89.

[23] D. Hensley and I. Richards, On the incompatibility of two conjectures concerning primes, Proc. Symp. Pure Math. 24 (1973), 123-127.

[24] Kenneth Ireland and Michael Rosen, A classical introduction to modern number theory, Springer Verlag N.Y., 1990.

[25] H Maier, Primes in short intervals, Michigan Mathematical Journal (1985).

[26] H.L. Montgomery and R.C. Vaughan, The large sieve, Mathematika Journal 40 (1973), 119134 . 
[27] Thomas R. Nicely, Some results of computational research in prime numbers (Computational number theory), 2009. http://www.trnicely.net.

[28] _ Skewes' problem, 2009. http://www.trnicely.net.

[29] John Nicholson, Nicholson's conjecture, 2013. https://oeis.org/A182514.

[30] János Pintz, Very large gaps between consecutive primes, Journal Of Number Theory (1997).

[31] Robert A. Rankin, The difference between consecutive prime numbers, Journal London Mathematical Society (1938).

[32] J.B. Rosser and L. Schoenfeld, Approximate formulas for some functions of prime numbers, Illinois Journal of Mathematics 6 (1962), 64-94.

[33] Sharper bounds for the Chebyshev functions $\theta_{x}$ and $\psi_{x}$, Mathematics of Computation 29 (1975), no. 129, 243-269.

[34] Sebastian M. Ruiz, A result on prime numbers, Mathematical Gazette 81 (1997), no. 269, 269.

[35] Atle Selberg, On the normal density of primes in small intervals and the difference between consecutive primes, Arch. Mathem. B 47 (1943), 87-105.

[36] _ An elementary proof of the prime number theorem, Ann. Math. 50 (1949), 305-313.

[37] Daniel Shanks, On maximal gaps between successive primes, Math. Comp. 18 (1964), 646651.

[38] Tomas Oliveira e Silva, Gaps between consecutive primes, 2006. www.ieeta.pt/ tos/gaps.html.

[39] K. Soundararajan, Small gaps between prime numbers: the work of Goldston-Pintz-Yildirim, Bulletin of the American Mathematical Society (2007).

[40] E. Westzynthius, Über die Differenzen Verteilung der Zahlen die zu den $n$ ersten Primzahlen teilerfremd sind, Comm. Phys. Math. Soc. Sci. Fenn. 5 (1931), 1-37. 


\section{APPENDIX}

This section contains the theory cited in the text, proofs of which are given in the provided references. Theorems 3.1 and 3.2 are stated in a slightly relaxed form, after the Floor function had been dropped:

Theorem 3.1 (Maximal Prime Gaps Bound (Feliksiak [11])).

For any $n \in \mathbb{N} \mid n \geq 8$ there exists at least one $p \in \mathbb{N} \mid n<p \leq n+\mathcal{G}_{(n)}=t$; where $p$ is as usual a prime number and the maximal prime gaps upper bound $\mathcal{G}_{(n)}$ is given by:

$$
\begin{array}{r}
\mathcal{G}_{(n)}=5\left(\log _{10} n\right)^{2} \quad \forall n \in \mathbb{N} \mid n \geq 8 \\
\text { Equivalently, } p_{i+1}-p_{i} \leq \mathcal{G}_{\left(p_{i}\right)}
\end{array}
$$

Theorem 3.2 (Maximal Prime Gaps Supremum Bound (Feliksiak [12])).

For any $n \in \mathbb{N} \mid n \geq 11$ there exists at least one $p \in \mathbb{N} \mid n<p \leq n+\mathcal{G} s_{(n)}=t$; where $p$ is as usual a prime number and the maximal prime gaps standard measure $\mathcal{G} s_{(n)}$ is given by:

$$
\begin{aligned}
c=\mathcal{G} s_{(n)}=5\left(\log _{10} n\right)^{2}-\frac{15}{8}\left(\log _{10} n\right) \quad \forall n \in \mathbb{N} \mid n \geq 11 \\
\text { Equivalently, } p_{i+1}-p_{i} \leq \mathcal{G} s_{\left(p_{i}\right)}
\end{aligned}
$$

Definition 3.3 (Alpha Constant). $\alpha=\left(\frac{\left(5+(\log 10)^{2}\right)}{(\log 10)^{2}}\right)$

Definition 3.4 (Beta Constant). $\beta=\left(\frac{(\exp 1) \pi}{2}\right)$

Theorem 3.5 (Maximal Prime Gaps Lower Bound (Feliksiak [14])).

The maximal prime gaps Lower Bound, for all $p_{(n)} \in \mathbb{N} \mid p_{(n)} \geq 23$ is given by:

$$
\mathfrak{g}=p_{(n+1)}-p_{(n)} \geq \mathcal{L} \mathcal{B}_{p_{n}}=\mathcal{M} \times\left(5\left(\log _{10} p_{n}\right)^{\alpha}-7\left(\log _{10} p_{n}\right)^{(2 \gamma)}\right)+\beta
$$

Where $\alpha$ and $\beta$ are given by the Definitions 3.3 and 3.4 respectively, $\gamma$ is the EulerMascheroni constant and $\mathcal{M}$ is given by:

$$
\mathcal{M}=\left(1-\frac{\sqrt{5}-1}{\log _{10} p_{n}}\right) \text {. }
$$

Lemma 3.6 (Upper Bound on the logarithm of the primorial).

The natural logarithm of the primorial function is strictly less than the respective prime number $p \in \mathbb{N}$ :

(3.5) $\log p_{(n)} \sharp<p_{(n)} \leq n \quad \forall n \in \mathbb{N} \mid n \geq 2$, where $p_{n}$ is the largest prime $p \leq n$ In particular the natural logarithm of the primorial function is asymptotic (from below) to the respective prime number:

$$
\log p_{(n)} \sharp \sim p_{(n)}
$$


Lemma 3.7 (Lower Estimation Error Bound On The Difference $\left.p_{n}-\log p_{n} \sharp\right)$.

The error of estimation of the primorial function by the use of the value of $p_{(n)}$ imposes the following lower bound:

$$
\begin{gathered}
\mathcal{L B}_{p_{(n)}}=(\sqrt{5}-1)\left(4 \gamma^{2}-2 \gamma\right)\left(\log p_{(n)}\right) \sqrt[3]{p_{(n)}}<\left(p_{(n)}-\log p_{(n)} \sharp\right) \\
\forall p_{(n)} \in \mathbb{N} \mid p_{(n)} \geq 2
\end{gathered}
$$

where $\gamma \approx 0.57721566490153286060651209$ is the Euler-Mascheroni constant.

Lemma 3.8 (Upper Estimation Error Bound On The Difference $\left.p_{n}-\log p_{n} \sharp\right)$.

The error of estimation of the primorial function by the use of the value of $p_{(n)}$ imposes the following upper bound:

$$
\left(p_{(n)}-\log p_{(n)} \sharp\right)<2 \sqrt{p_{(n)}}=\mathcal{U} \mathcal{B}_{p_{(n)}} \quad \forall p_{(n)} \in \mathbb{N} \mid p_{(n)} \geq 2
$$

Regarding the proofs of Lemmas 3.6, 3.7 and 3.8, please consult Feliksiak [11].

Theorem 3.9 (The Supremum Bound of the sum of $\left.(\log p)^{2}\right)$. by:

The Supremum of the sum of $(\log p)^{2}$ terms, for all $p_{n} \in \mathbb{N} \mid p_{(n)} \geq 3$ is given

$$
\text { Sup }=\left(\left(p_{n}^{(-G M)}+1\right)\left(\log p_{n}-1\right) \log p_{n} \sharp\right)+\frac{\pi}{2} \geq \sum_{p \leq n}(\log p)^{2}
$$

Where $G M=\left(\frac{\sqrt{5}-1}{2}\right)$ is the Golden Mean constant.

Lemma 3.10 (Relative Error $\mathcal{R} . \mathcal{E} \cdot{ }_{\left(p_{n}\right)}$ of the Infimum of the sum $\left.\sum_{p \leq n}(\log p)^{2}\right)$.

The relative error of the Infimum for all $p_{n} \in \mathbb{N} \mid p_{(n)} \geq 3$ has the following bounds:

$$
\mathcal{L B}=\frac{1}{\sqrt{5} \sqrt[\beta 1]{p_{n}}}<\frac{\sum_{p \leq n}(\log p)^{2}-\left[\operatorname{Inf}=\left(\left(\log p_{n}-1\right) \log p_{n} \sharp\right)+\log 2\right]}{\sum_{p \leq n}(\log p)^{2}}<\frac{\alpha}{G M \sqrt[\beta 2]{p_{n}}}=\mathcal{U B}
$$

Where $\alpha=\frac{5}{(\log 10)^{2}}, \beta 1=(\sqrt{5}-1), \beta 2=(\log 2+1)$ and $G M=\left(\frac{\sqrt{5}-1}{2}\right)$.

Lemma 3.11 (Upper bound on the $\left.\operatorname{sum} \sum_{p \leq n}(\log p)^{2}\right)$. by:

The Upper bound on the sum $\sum_{p \leq n}(\log p)^{2}$ for all $p_{n} \in \mathbb{N} \mid p_{(n)} \geq 11$ is given

$$
U B_{p_{n}}=\left(\log p_{n}-1\right) p_{n}+1
$$


Lemma 3.12 (Lower bound on the sum $\left.\sum_{p \leq n}(\log p)^{2}\right)$. by:

The Lower bound on the sum $\sum_{p \leq n}(\log p)^{2}$, for all $p_{n} \in \mathbb{N} \mid p_{(n)} \geq 2$ is given

$$
L B_{p_{n}}=\left(\log p_{n}-1\right)\left(p_{n}-2 \sqrt{p_{n}}\right)
$$

Regarding the proofs of Theorem 3.9 and Lemmas 3.10 through 3.12, please consult Feliksiak [17].

Definition 3.13 (Stepwise lower integration limit). $\theta_{1}=\log p_{(i)} \sharp$

Definition 3.14 (Stepwise upper integration limit). $\theta_{2}=\log p_{(i+1)} \sharp$

Definition 3.15 (Step sequence).

$$
\mathcal{S S \mathcal { Q } _ { ( p _ { ( i + 1 ) } ) }}=\left(T L i_{\left(p_{(i+1)}\right)}-T L i_{\left(p_{i}\right)}\right)=\int_{\theta_{1}}^{\theta_{2}} \frac{d t}{\log t} \quad \forall p_{i} \in \mathbb{N} \mid p_{i} \geq 3
$$

Definition 3.16 (Step estimation error sequence).

$$
\mathcal{S E R}_{\left(p_{(i+1)}\right)}=\left(T L i_{\left(p_{(i+1)}\right)}-T L i_{\left(p_{i}\right)}-1\right)=\int_{\theta_{1}}^{\theta_{2}} \frac{d t}{\log t}-1 \quad \forall p_{i} \in \mathbb{N} \mid p_{i} \geq 3
$$

Lemma 3.17 (Stepwise Convergence Of The Error of Estimation of the $T L i_{(n)}$ ). The step sequence of the tailored logarithmic integral $T L i_{(n)}$ is Cauchy and converges asymptotically from above to the limit:

$$
\lim _{p_{i} \rightarrow \infty}\left(\int_{\theta_{1}}^{\theta_{2}} \frac{d t}{\log t}\right)=1
$$

Furthermore, the difference of the step integral $T L i_{(n)}$ and its approximation has the following bounds:

$$
\begin{aligned}
L B_{p_{(i+1)}}=\frac{1}{5\left(p_{(i+1)}\right)} \leq\left[\int_{\theta_{1}}^{\theta_{2}} \frac{d t}{\log t}-\frac{\log p_{(i+1)}}{\log \left(\log p_{(i+1)} \sharp\right)}\right] \leq & \frac{1}{p_{(i+1)}}=U B_{p_{(i+1)}} \\
& \text { for all } p \in \mathbb{N} \mid p \geq 13
\end{aligned}
$$

with $\theta_{1}$ and $\theta_{2}$ given by the Definitions 3.13 and 3.14 respectively.

Theorem 3.18 (The Step Sequence Estimation Error Lower Bound).

The estimation error of the tailored logarithmic integral TLi $i_{(n)}$ at every step exceeds the value of the inverse of the pertinent prime number hence, it is bounded below by $1 / p \forall p \in \mathbb{N} \mid p_{i} \geq 13$ :

$$
\operatorname{SER}_{\left(p_{(i+1)}\right)}=\left(\int_{\theta_{1}}^{\theta_{2}} \frac{d t}{\log t}\right)-1>\frac{1}{p_{(i+1)}}
$$

where $p_{(i)}$ and $p_{(i+1)}$ are associated with the lower/upper limit of integration $\theta_{1}$ and $\theta_{2}$ respectively. 
We need to re-define the lower/upper limits of integration to conform with the summation limits. The computation of the sum of step errors of the integral $T L i_{n}$ begins at $p_{2}=3$, irrespective of the fact that the computation of the sums pertinent to the bounds (Infimum, Supremum, Lower and Upper) begins first at $p_{15}=47$.

Definition 3.19 (Theta applicable for summation). $\theta_{1}=\log \left(p_{(2+(k-1))} \sharp\right)$

Definition 3.20 (Theta applicable for summation). $\theta_{2}=\log \left(p_{(2+k) \sharp)}\right.$

Theorem 3.21 $\left(T L i_{(n)}\right.$ Estimation Error Divergence).

The error arising in the estimation of the prime counting function $\pi_{(n)}$ by the application of the tailored logarithmic integral $T L i_{(n)}$, diverges to infinity:

$\lim _{n \rightarrow \infty}\left(T L i_{(n)}-\pi_{(n)}\right)=\lim _{p_{(n)} \rightarrow \infty}\left(\sum_{(k=1)}^{\left(\pi_{(n)}-2\right)}\left\{\left(\int_{\theta_{1}}^{\theta_{2}} \frac{d t}{\log t}\right)-1\right\}+\left(\int_{2}^{\log 6} \frac{d t}{\log t}-\pi_{3}\right)\right) \rightarrow \infty$

Where the limits of integration $\theta_{1}$ and $\theta_{2}$ are given by the Definitions 3.19 and 3.20 respectively. Besides, the prime number $p_{(n)}$ is defined as being the biggest prime $p \leq n$. Furthermore,

$$
\begin{gathered}
\left(T L i_{(n)}-\pi_{(n)}\right)>\sum_{p \leq n} \frac{1}{p} \forall n \in \mathbb{N} \mid n \geq 983 \\
T L i_{(n)}=\int_{2}^{\theta_{(n)}} \frac{d t}{\log t}>\pi_{(n)} \quad \forall n \in \mathbb{N} \mid n \geq 43
\end{gathered}
$$

Regarding the proofs of Lemma 3.17 and Theorems 3.18 and 3.21, please consult Feliksiak [11]. 